\title{
Metamorphoses: Sudden Jumps in Basin Boundaries *
}

\author{
Kathleen T. Alligood ${ }^{1}$, Laura Tedeschini-Lalli ${ }^{2}$, and James A. Yorke ${ }^{3}$ \\ 1 Department of Mathematical Sciences, George Mason University, Fairfax, VA 22030, USA \\ 2 Dipartimento di Matematica, Universita di Roma "La Sapienza", I-00185 Rome, Italy \\ 3 Department of Mathematics and, Institute for Physical Science and Technology, \\ University of Maryland, College Park, MD 20742, USA
}

Received August 14, 1986; in revised form April 15, 1990

\begin{abstract}
In some invertible maps of the plane that depend on a parameter, boundaries of basins of attraction are extremely sensitive to small changes in the parameter. A basin boundary can jump suddenly, and, as it does, change from being smooth to fractal. Such changes are called basin boundary metamorphoses. We prove (under certain non-degeneracy assumptions) that a metamorphosis occurs when the stable and unstable manifolds of a periodic saddle on the boundary undergo a homoclinic tangency.
\end{abstract}

Dynamical systems in the plane can have many coexisting attractors. In order to be able to predict long-term or asymptotic behavior in such systems, it is important to be able to recognize to which attractor (final state) a given trajectory will tend. The set of initial conditions whose trajectories are asymptotic to a particular attractor is called the basin of attraction of that attractor. In some systems that depend on a parameter, it has been observed that the boundaries of these basins are extremely sensitive to small changes in the parameter. Not only can a boundary jump suddenly, but it can also change from being smooth to being fractal. These changes, called boundary metamorphoses, are studied at length in [GOY]. In this paper we prove a theorem, originally stated in [GOY], which characterizes the jumps in basin boundaries.

The Hénon map $f(x, y)=\left(A-x^{2}-J y, x\right)$ provides an example of this phenomenon. We fix $J=0.3$ and vary $A$, resulting in a one-parameter, invertible map of the plane. The Jacobian of $f$ is $J$; hence, $f$ is area contracting for all $A$. We will be looking specifically at the boundary of the basin of attraction of infinity. (The basin of infinity is the set of all points $(x, y)$ such that $\left|f^{n}(x, y)\right| \rightarrow \infty$ as $n \rightarrow \infty$.) Figures 1a and $1 \mathrm{~b}$ show the basin of infinity in black for $A=1.314$ and $A=1.320$, respectively. In Fig. $1 \mathrm{~b}$ we see that the basin of infinity contains points which were previously (at $A=1.314$ ) well within the white region. This new set of black points has not

* This research was supported in part by grants and contracts from the Defense Advanced Research Projects Agency, the Consiglio Nazionale delle Ricerche(Comitato per le Matematiche), and the National Science Foundation 

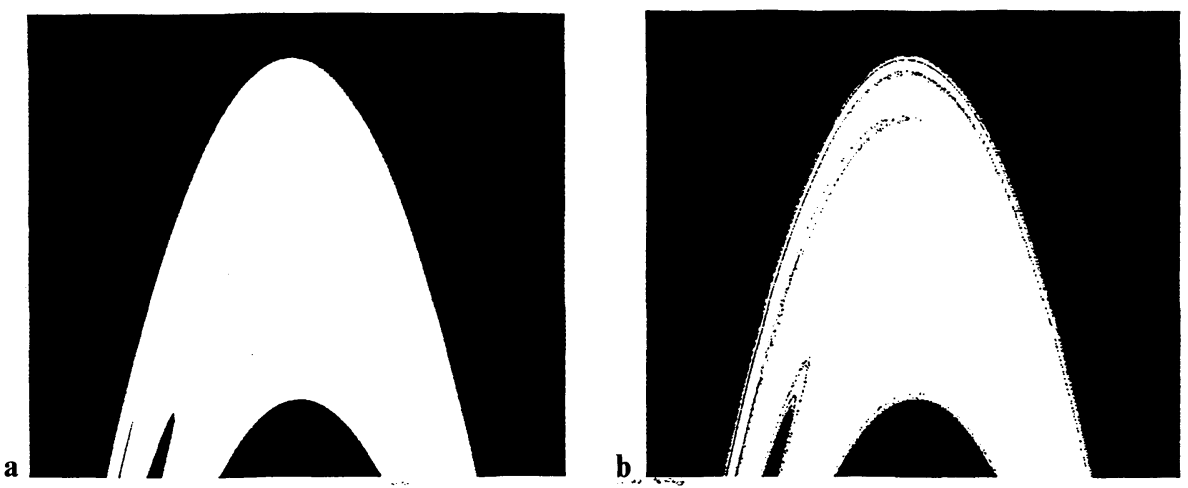

Fig. 1a and b. The figure shows the basin of attraction of infinity in black for the Henon map

$$
f(x, y)=\left(A-x^{2}-J y, x\right) .
$$

We fix $J=0.3$ a $A$ is 1.314 , and in b $A$ is increased to 1.32 . The change in the basin of infinity illustrates a basin boundary jump

gradually moved in from the boundary of the white region. Rather, beyond a certain critical value $A=A^{*} \approx 1.3145$, black points suddenly begin appearing deep in the interior of the white region. As $A$ increases, the thin bands thicken. This is a discontinuous change in the basin of infinity.

In order to understand this phenomena, we must examine the dynamical behavior on the basin boundary. At $A=1.314$ (Fig. 1a) the boundary is observed numerically to consist of a saddle fixed point $p_{1}$, and its stable manifold $W^{s}\left(p_{1}\right)$. (The stable manifold $W^{s}(p)$ of a fixed point $p$ is the set of points $(x, y)$ such that $f^{n}(x, y) \rightarrow p$ as $n \rightarrow \infty$. More generally, the stable manifold $W^{s}\left(p_{k}\right)$ of a periodic point $p_{k}$ of period $k$ is the set of points $(x, y)$ such that $f^{n k}(x, y) \rightarrow p_{k}$ as $n \rightarrow \infty$. Analogously, the unstable manifold $W^{u}\left(p_{k}\right)$ of $p_{k}$ is the set of points $(x, y)$ such that $f^{-n k}(x, y) \rightarrow p_{k}$ as $n \rightarrow \infty$. Such sets can be proved to be smooth curves.) One branch of the unstable manifold of $p_{1}$ at $A=1.314$ extends into the white region, as shown in Fig. 2a. At the critical value $A^{*} \approx 1.3145$, after which the basin boundary jumps into the white region, we find that $W^{s}\left(p_{1}\right)$ and $W^{u}\left(p_{1}\right)$ are tangent (Fig. 2b). Hammel and Jones $[\mathrm{HJ}]$ were the first to prove a theorem relating the tangency of $W^{s}\left(p_{1}\right)$ and $W^{u}\left(p_{1}\right)$ (called a homoclinic tangency) to basin boundary metamorphoses. Their methods are different from ours, however. We want to relate these metamorphoses to the saddle periodic orbits which are found near the points of tangency and which we describe below.

The complicated dynamical behavior which occurs at homoclinic tangencies has been studied at length in recent years, especially in the papers of Gavrilov and Silnikov [GS], Newhouse [N], and Robinson [R]. Under certain non-degeneracy assumptions, there are horseshoe maps defined on subsets of the plane near a point $q_{0}$ of tangency of $W^{s}\left(p_{1}\right)$ and $W^{u}\left(p_{1}\right)$. Figure 3 shows a rectangle $B_{4}$ and some of its iterates under $f$. Notice that $f^{4}\left(B_{4}\right)$ is horseshoe-shaped and intersects $B_{4}$ in two components. In fact, for $n$ sufficiently large, there is a rectangle $B_{n}$ near the point of tangency $q_{0}$ such that $f^{n}$ restricted to $B_{n}$ is a horseshoe map. There is necessarily a saddle orbit of period $n$ in each of the two components of the intersection of $B_{n}$ and $f^{n}\left(B_{n}\right)$ (see, for example, [R]). One of these saddles will have a "flipped" unstable manifold (i.e., $D_{x} f^{n}$ at this saddle has an eigenvalue less than -1 ), and the other 
a
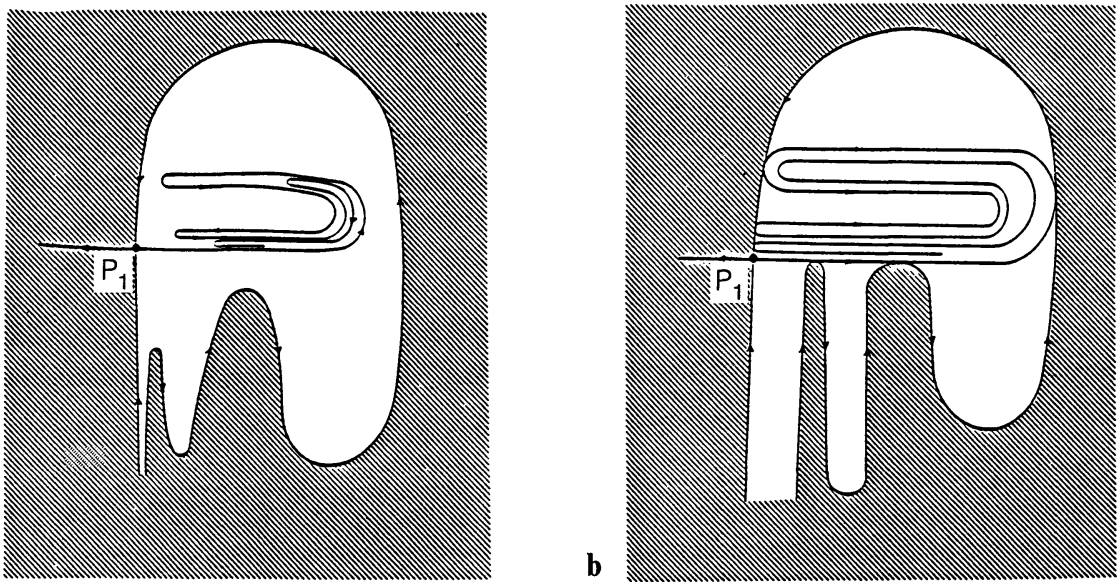

Fig. 2a and $\mathbf{b}$. The figure show the stable and unstable manifolds of a fixed point $p_{1}$ before and at tangency, respectively

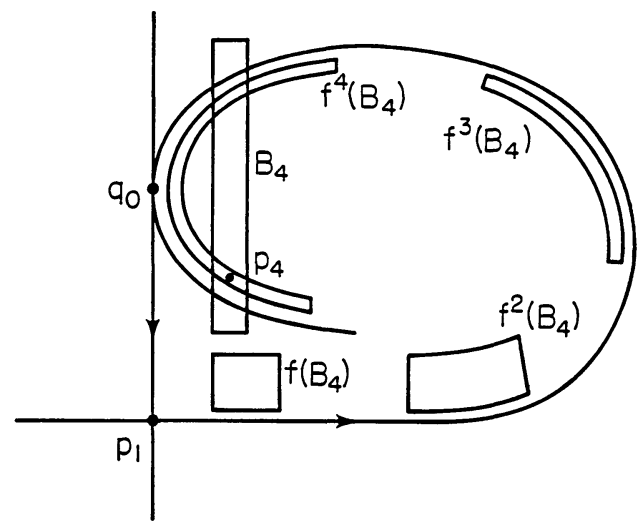

Fig. 3. This illustrates a horseshoe map. The invariant set of the horseshoe is in $B_{4} \cap f^{4}\left(B_{4}\right)$

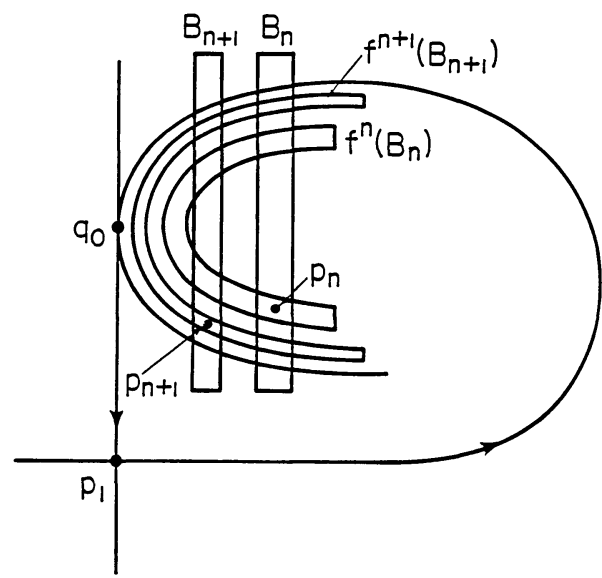

Fig. 4. It shows the relative positions of two simple Newhouse saddles $p_{n}$ and $p_{n+1}$ of periods $n$ and $n+1$, respectively 
will not. We label the unflipped saddle $p_{n}$. This orbit is called a "simple Newhouse saddle" in [TY].

The larger $n$ is, the closer $B_{n}$ will be to $q_{0}$ and $W^{s}\left(p_{1}\right)$. This corresponds to the fact that the length of time (i.e., the number of iterates of $f$ ) it takes for a point to move around the fixed point $p_{1}$ is determined by how close the point is to the stable manifold $W^{s}\left(p_{1}\right)$. What we see (Fig. 4) is an infinite family of horseshoes, and a sequence $\left\{p_{n}\right\}$ of simple Newhouse saddles (where $p_{n}$ has period $n$ and is in $B_{n}$ ) such that $\left\{p_{n}\right\} \rightarrow q_{0}$. In the following theorem, as stated in [GOY], the saddle fixed point $S$ corresponds to $p_{1}$ in the discussion above, and the saddle orbit $T$ corresponds to a simple Newhouse saddle $p_{n}$, for some $n$. The term "first non-degenerate tangency" refers to the following set $(H)$ of hypotheses:

(i) $W^{u}\left(p_{1}\right)$ does not intersect $W^{s}\left(p_{1}\right)$ for $A<A_{*}$.

(ii) When $A=A_{*}, W^{u}(p)$ forms a tangency of "finite order" with $W^{s}(p)$ at a point $q_{0}$; i.e., there is a coordinate system $(u, v)$ in a neighborhood of $q_{0}$ such that $q_{0}$ corresponds to $(0,0), W^{s}(p)$ corresponds to the $u$-axis $(v=0)$, and $W^{u}(p)$ corresponds to $\{(u, v): v=h(u)\}$, where $\frac{d^{i} h}{d u^{i}}=\left\{\begin{array}{ll}0 & \text { if } 1 \leqq i<n \\ a>0 & \text { if } i=n\end{array}\right.$ for some $n \geqq 2 ;$ and (iii) $W^{u}(p)$ crosses $W^{s}(p)$ with non-zero speed. I.e., for each $A$ in $\left(A_{*}, A_{*}+\delta\right), W^{u}(p)$ intersects $W^{s}(p)$ at a point $q_{A}$ (the continuation of $q_{0}$ ). Furthermore, for each neighborhood $\mathscr{U}$ of $q_{0}$ sufficiently small that $\mathscr{U}-W^{s}(p)$ is disconnected, there are points of $W^{u}\left(p_{1}\right)$ in both components of $\mathscr{U}-W_{A}^{s}\left(p_{1}\right)$, where $W_{A}^{s}\left(p_{1}\right)$ is the component of $\mathscr{U} \cap W^{s}\left(p_{1}\right)$ containing $q_{A}$.

For the application in which $W^{s}\left(p_{1}\right)$ is the boundary of the basin of infinity, condition (iii) implies that points in $W^{u}\left(p_{1}\right)$ are in the basin of infinity for each $A$ in $\left(A_{*}, A_{*}+\delta\right)$.

Theorem. Consider a diffeomorphism $f$ of the plane depending on a parameter $A$ with a saddle fixed point or periodic orbit $S$. We assume that the absolute value of the determinant of the Jacobian of $f$ ( or of $f^{n}$ in the case of a periodic orbit of period $n$ ) is less than one at every point of the plane. Assume that $f$ has a transition value $A_{*}$ as $A$ increases where the stable and unstable manifolds of $S$ have a non-degenerate tangency and then cross for the first time. Then there will be a periodic saddle $T$ that is in the closure of the stable manifold of $S$ for all $A$ slightly greater than $A_{*}$ but is not in it at $A_{*}$. The saddle $T$ is a positive distance from the stable manifold of $S$ for $A=A_{*}$.

We prove this theorem with the aid of the following lemma. The existence of the sequence $\left\{p_{n}\right\}$ of hyperbolic saddles was first demonstrated in the case of quadratic tangency by Gavrilov and Silnikov [GS], and later by techniques of Robinson [R] where arbitrary finite order tangencies are considered. For $n$ sufficiently large, the horseshoe map $f^{n}$ restricted to $B_{n}$ persists for parameter values beyond tangency, i.e., for $A$ in the interval $\left[A_{*}, A_{*}+\delta\right)$, where $\delta>0$ is very small. We let $p_{k}=p_{k}(A)$ be the continuation of the simple Newhouse saddle of period $k$ for $A$ in $\left[A_{*}, A_{*}+\delta\right.$ ).

Lemma. Let $p_{k}$ be a simple Newhouse saddle of period $k$ (as described above) for $A$ in $\left[A_{*}, A_{*}+\delta\right)$.

(i) There exists $N_{0}>0$ independent of $A$ in $\left[A_{*}, A_{*}+\delta\right)$ such that $W^{u}\left(p_{n}\right)$ crosses $W^{s}\left(p_{n+1}\right)$ for all $n \geqq N_{0}$.

(ii) For each $A$ in $\left(A_{*}, A_{*}+\delta\right)$ there exists $N_{1}(A) \geqq 0$ such that $W^{u}\left(p_{m}\right)$ crosses $W^{\mathrm{s}}\left(p_{1}\right)$, for all $m \geqq N_{1}(A)$. 


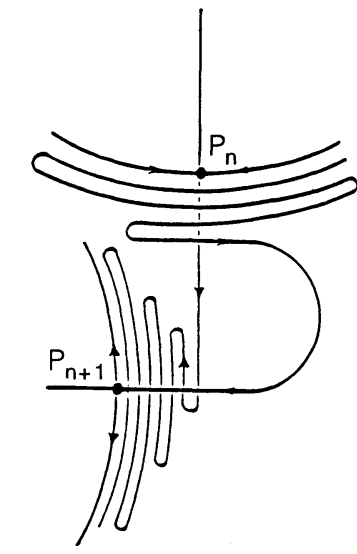

a

Fig. 5a and b. This indicates that the closure of $W^{u}\left(p_{n+1}\right)$ is contained in the closure of $W^{u}\left(p_{n}\right)$. b indicates that the point of tangency $q_{0}$ is in the closure of the unstable manifolds of infinity many simple Newhouse saddles

We postpone the proof of this lemma due to its technical nature and proceed to show how the theorem follows. By part (i) of the lemma, we assume that there exists $N_{0}>0$ independent of $A$ in $\left[A_{*}, A_{*}+\delta\right)$ such that $W^{u}\left(p_{n}\right)$ crosses $W^{s}\left(p_{n+1}\right)$ for all $n \geqq N_{0}, A$ in $\left[A_{*}, A_{*}+\delta\right)$. The crossing of $W^{u}\left(p_{n}\right)$ and $W^{s}\left(p_{n+1}\right)$ implies that the forward iterates of any segment of $W^{u}\left(p_{n}\right)$ containing $x$ will eventually contain all of $\overline{W^{u}\left(p_{n+1}\right)}$ (the closure of $\left.W^{u}\left(p_{n+1}\right)\right)$ in its set of limit points (see Fig. 5a). For a discussion of this result in the case of a transverse crossing (the $\lambda$-Lemma), see, for example, the exposition in [GH]. Hence $\overline{W^{u}\left(p_{n+1}\right)} \subset \overline{W^{u}\left(p_{n}\right)}$. Proceeding inductively, we have

(i) $\overline{W^{u}\left(p_{m}\right)} \subset \overline{W^{u}\left(p_{n}\right)}$, (see Fig. 5b), for every $m \geqq n, n \geqq N_{0}$. By part (ii) of the lemma, there exists a number $N_{1}(A)>0$ such that $W^{u}\left(p_{m}\right)$ crosses $W_{1}^{s}(p)$ for $m_{1} \geqq N(A)$, $A \in\left(A_{*}, A_{*}+\delta\right)$. Thus

(ii) $\overline{W^{u}\left(p_{1}\right)} \subset \overline{W^{u}\left(p_{m}\right)}$, for every $m \geqq N_{1}(A), A \in\left(A_{*}, A_{*}+\delta\right)$. Putting together (i) and (ii), we have

(iii) $\overline{W^{u}\left(p_{1}\right)} \subset \overline{W^{u}\left(p_{n}\right)}$, for all $n \geqq N_{0}, A \in\left(A_{*}, A_{*}+\delta\right)$. For $A>A_{*}, W^{u}\left(p_{1}\right)$ crosses $W^{s}\left(p_{1}\right)$. By (iii), $W^{u}\left(p_{n}\right)$ must also cross $W^{s}\left(p_{1}\right)$, for all $n \geqq N_{0}$. Thus $\overline{W^{s}\left(p_{n}\right)} \subset \overline{W^{s}\left(p_{1}\right)}$, for all $n \geqq N_{0}$, and $p_{N_{0}}$ is in the closure of $W^{s}\left(p_{1}\right)$, for all $A$ in $\left[A_{*}, A_{*}+\delta\right.$ ).

\section{Remarks.}

(1) At $A=A_{*}$, each of the saddles $p_{n}$ is a positive distance from the boundary $W^{s}\left(p_{1}\right)$ of the basin of infinity. For every $A$ slightly larger than $A_{*}$, the theorem says that there is an $N_{0}$ such that $p_{N_{0}}$ is in the closure of $W^{s}\left(p_{1}\right)$. Thus there is a jump in the boundary at $A=A_{*}$.

(2) Since the Hénon map is analytic, the tangency at $A_{*} \approx 1.314(J=0.3)$ is of finite order and techniques of [R] would apply. At this tangency $N_{0}$ appears to be 4 (see [GOY]). This is supported by computer evidence that for $A$ slightly greater than 1.314 , the saddle $p_{4}$ is on the boundary of the basin of infinity.

(3) The proof of the theorem characterizes the boundary after tangency by showing that there are infinitely many saddles and their stable manifolds contained in $\overline{W^{s}\left(p_{1}\right)}$. The fact that there is a jump in the boundary is, of course, 
implied by this characterization. The existence of such a jump can be demonstrated by a simpler, topological argument. For any path $I$ connecting the left and right sides of $B_{n}$ (cf. Fig. 4), $f^{n}(I)$ extends through the horseshoe image $f^{n}\left(B_{n}\right)$. If $f^{n}\left(B_{n}\right)$ crosses $B_{n+1}$ (as shown in Fig. 4), a portion of $f^{n}(I)$ connects the left and right side of $B_{n+1}$. If, at tangency $\left(A=A_{*}\right), f^{r}\left(B_{r}\right)$ so crosses $B_{r+1}$ for all $r, r \geqq n$, then $\bigcup_{r \geqq n} f^{r}(I)$ contains $q_{0}$. For $A>A_{*}$, some forward iterate of $I$ will then cross $W^{s}\left(p_{1}\right)$.

Proof of Lemma. Following the construction of [R, TY, and GH] (Sect. 6.6), we assume the following:

(i) $D F\left(p_{1}\right)$ has eigenvalues $v$ and $\lambda$ which satisfy $0<v<1, \lambda>1$, and $v \lambda<1$, for all $A$ near $A_{*}$.

(ii) There exists a neighborhood $U$ of $p_{1}$ in which the map $f$ is linear up to smooth changes of coordinates; i.e., $f(x, y, A)=(\lambda x, v y)$ for $(x, y)$ in $U$, all $A$ near $A_{*}$. (Here we need an additional non-resonance assumption - namely, that $v$ and $\lambda$ are not integer multiples of each other.) Hence, locally, $W^{s}\left(p_{1}\right)$ is given by the $y$-axis and $W^{u}\left(p_{1}\right)$ is given by the $x$-axis.

(iii) There is a non-degenerate tangency of $W^{s}\left(p_{1}\right)$ and $W^{u}\left(p_{1}\right)$. Specifically, there exist points $\left(p_{0}, 0\right)$ in $W^{u}\left(p_{1}\right) \cap U$ and $\left(0, q_{0}\right)$ in $W^{s}\left(p_{1}\right) \cap U$ such that $f^{k}\left(p_{0}, 0, A_{*}\right)$ $=f_{A^{*}}^{k}\left(p_{0}, 0\right)=\left(0, q_{0}\right)$ and $W^{s}\left(p_{1}\right)$ and $W^{u}\left(p_{1}\right)$ near $\left(0, q_{0}\right)$ satisfy $(H)$. Let $V=\left[p_{0}-\varepsilon\right.$, $\left.p_{0}+\varepsilon\right] \times[0, \varrho]$ for some $\varepsilon>0$ and $\varrho>0$, and let $W$ be a trapezoidal neighborhood with vertices $f_{A^{*}}^{k}\left(p_{0}-\varepsilon, 0\right), f_{A^{*}}^{k}\left(p_{0}+\varepsilon, 0\right)$, and the projections of these points on the $y$-axis, $W^{s}\left(p_{1}\right)$. (See Fig. 6.) We assume that $V$ and $W$ are in $\mathscr{U}$.

For $n$ sufficiently large and $A=A_{*}, f^{-n+k}(V)$ stretches across $W$. For such $n$, let $B_{n}=f^{-n+k}(V) \cap W$. Actually, since $f^{-n+k}(V)$ may wind around a lot, we let $B_{n}$ be the connected component of $f^{-n+k}(V) \cap W$ which is nearest $W^{s}\left(p_{1}\right)$. Under hypotheses $(H)$, we know that $f^{n}$ restricted to $B_{n}$ is a horseshoe map, in the sense of Smale [S]. (See, for example, [GH] in the case of quadratic tangency and [R] for the case of finite order tangency.) Specifically, we use the following facts about such maps:

(1) $B_{n}$ and $f^{n}\left(B_{n}\right)$ intersect in two components, $W_{1, n}$ and $W_{2, n}$. The saddle $p_{n}$ is contained in $W_{1, n}$ and is the only fixed point of $f^{n}$ in $W_{1, n}$. Furthermore, $p_{n}$ is the only point in $W_{1, n}$ which stays in $W_{1, n}$ under all forward and backward iterates of $f^{n}$.

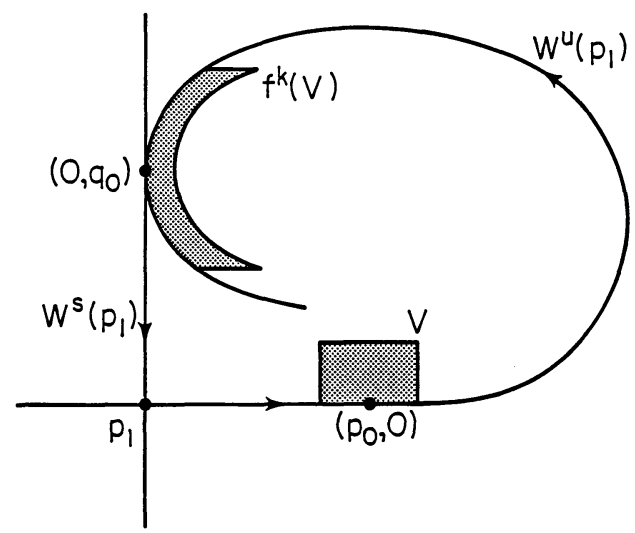

Fig. 6. The figure illustrates definitions used in the proof of the Lemma 


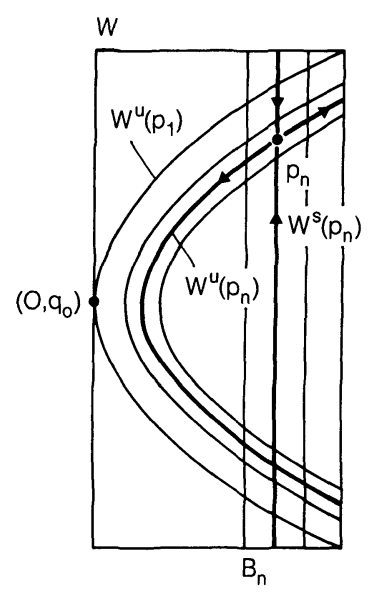

Fig. 7. It shows parts of the stable and unstable manifolds of the simple Newhouse saddle $p_{n}$

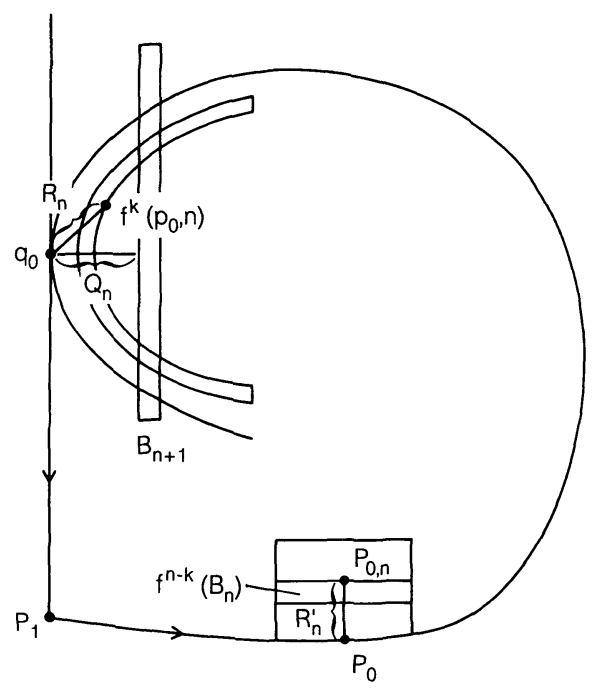

Fig. 8. This illustrates definitions used in the proof of the Lemma

(2) The only points which stay in $W_{1, n}$ under all forward (respectively, backward) iterates of $f^{n}$ are in $W^{s}\left(p_{n}\right)$ (respectively $W^{u}\left(p_{n}\right)$ ).

We argue that the stable manifold of $p_{n}$ extends (vertically) through $B_{n}$ (see Fig. 7). Let $L_{0}$ be any horizontal segment in $B_{n}$. It is easily seen that $f^{n}\left(L_{0}\right)$ is a parabola which extends through $f^{n}\left(B_{n}\right)$. Recursively, let $L_{i}=f^{n}\left(L_{i-1}\right) \cap W_{1, n}$ for $i=1,2,3, \ldots$. Then $f^{-n i}\left(L_{i}\right), i \geqq 1$, are nested intervals converging to a point $z_{0}$. Since $f^{m n}\left(z_{0}\right)$ is in $W_{1, n}$ for all $m>1, z_{0}$ must be in $W^{s}\left(p_{n}\right)$. This argument shows that $W^{s}\left(p_{n}\right)$ intersects the top and bottom of $B_{n}$ and first leaves $B_{n}$ through these sides. A similar argument (using iterates of $f^{-1}$ ) shows that $W^{u}\left(p_{n}\right)$ extends through the horseshoe $f^{n}\left(B_{n}\right)$, first leaving the horseshoe through the "feet." (See Fig. 7.)

In order to prove that $W^{u}\left(p_{n}\right)$ intersects $W^{s}\left(p_{n+1}\right)$, we will show that the horseshoe $f^{n}\left(B_{n}\right)$ containing $W^{u}\left(p_{n}\right)$ crosses through $B_{n+1}$ for $n$ sufficiently large 
(see Fig. 8). When $A=A_{*}$ let $Q$ be the distance from $\left(0, q_{0}\right)$ to $B_{n+1}$, let $R^{\prime}$ be the (vertical distance from $p_{0}$ to the point $p_{0, n}$ at the top of the rectangle $f^{n-k}\left(B_{n}\right)$, and let $R_{n}$ be the distance from $q_{0}=f^{k}\left(p_{0}\right)$ to $f^{k}\left(p_{0, n}\right)$, as shown in Fig. 8 .

By our assumption that $V$ and $W$ are in $U, Q$ is given by $\left(p_{0}-\varepsilon\right)\left(\lambda^{-(n-k+1)}\right)$, and $R_{n}^{\prime}$ is given by $\left(q_{0}+\delta\right) \mu^{(n-k)}$. Thus $\frac{R_{n}^{\prime}}{Q_{n}}=\frac{\lambda\left(q_{0}+\delta\right)}{\left(p_{0}-\varepsilon\right)}(\mu \lambda)^{(n-k)}$. Since $\mu \lambda<1, \frac{R_{n}^{\prime}}{Q_{n}} \rightarrow 0$ as $n \rightarrow \infty$. Similarly, $R_{n} / Q_{n} \rightarrow 0$ as $n \rightarrow \infty$, since $R_{n}$ is the distance from $q_{0}=f^{k}\left(p_{0}\right)$ to $f^{k}\left(p_{0, n}\right)$ and $f$, hence $f^{k}$, is Lipschitz; (i.e., there exists a constant $K$, independent of $n$, such that $\left.R_{n}=K R_{n}^{\prime}\right)$. Thus there exists $N_{0}>0$ such that $R_{n} / Q_{n}<1$ for $n \geqq N_{0}$. When $R_{n}<Q_{n}, W^{u}\left(p_{n}\right)$ crosses $W^{s}\left(p_{n+1}\right)$. Part (i) of the lemma follows from the observation that for $A \in\left[A_{*}, A_{*}+\delta\right)$, the horseshoe $f^{n}\left(B_{n}\right)$ is pulled even further through $B_{n+1}$ for $n \geqq N_{0}$, implying that $W^{u}\left(p_{n}\right)$ continues to cross $W^{s}\left(p_{n+1}\right)$.

For the proof of part (ii), notice that $f_{A}\left(p_{0, n}\right) \rightarrow f_{A}\left(p_{0}\right)$ for all $A$, since $p_{0, n} \rightarrow p_{0}$. For each $m>N_{0}$, the image $f_{A}^{m}\left(B_{m}\right)$ contains part of $W^{u}\left(p_{m}\right)$. Thus since $f_{A}\left(p_{0, m}\right)$ $\rightarrow f_{A}\left(p_{0}\right)$, there exists a sequence $\left\{r_{m}\right\}$ such that $r_{m}$ is on $W^{u}\left(p_{m}\right)$ and $r_{m} \rightarrow f_{A}\left(p_{0}\right)$. We assume without loss of generality that, for $A$ in $\left(A_{*}, A_{*}+\delta\right), f_{A}\left(p_{0}\right)$ is on the opposite side of $W^{s}\left(p_{1}\right)$ from $B_{n}$ and that the distance $\varrho(A)$ from $f_{A}\left(p_{0}\right)$ to $q_{0}$ is positive (by assumption (iii)). (Assumption (iii) does not say specifically that $f_{A}\left(p_{0}\right)$ is on the opposite side of $W^{s}\left(p_{1}\right)$, but that some points of $W^{u}\left(p_{1}\right)$ in a neighborhood of $f_{A}\left(p_{0}\right)$ are. The number $R_{n}^{\prime}$, however, measures the distance from $W^{u}\left(p_{1}\right)$ (the $x$-axis) to the rectangle $f^{n-k}\left(B_{n}\right)$ and thus is independent of the point $p_{0}^{\prime}$ chosen on $W^{u}\left(p_{1}\right)$ in a neighborhood of $p_{0}$.) Let $N_{1}(A)>0$ be sufficiently large that $\left|r_{m}-f_{A}\left(p_{0}\right)\right|$ $<\varrho(A)$, for all $m \geqq N_{1}(A)$. Then for $A \in\left(A_{*}, A_{*}+\delta\right), W^{u}\left(p_{m}\right)$ crosses $W^{s}\left(p_{1}\right)$ for all $m \geqq N_{1}(A)$.

\section{References}

[GH] Guckenheimer, J., Holmes, P.: Nonlinear oscillations, dynamical systems, and bifurcation of vector fields. Berlin, Heidelberg, New York: Springer 1983

[GOY] Grebogi, C., Ott, E., Yorke, J.: Basin boundary metamorphoses: Changes in accessible boundary orbits. Physica 24D, 243-262 (1987)

[GS] Gavrilov, N., Silnikov, L.: On three-dimensional dynamical systems close to systems with structurally unstable homoclinic curve I. Math. USSR Sbornik 17, 467-485 (1972); and Part II. Math. USSR Sbornik 19, 139-156 (1973)

[HJ] Hammel, S., Jones, C.: Jumping stable manifolds for dissipative maps of the plane. Physica 35D, 87-106 (1989)

[N] Newhouse, S.: Diffeomorphisms with infinitely many sinks. Topology 13, 9-18 (1974)

[R] Robinson, C.: Bifurcation to infinitely many sinks. Commun. Math. Phys. 90, 433-459 (1983)

[S] Smale, S.: Differentiable dynamical systems. Bull. Am. Math. Soc. 73, 747-817 (1967)

[TY] Tedeschini-Lalli, L., Yorke, J.: How often do simple dynamical processes have infinitely many sinks? Commun. Math. Phys. 106, 635-657 (1986) 